\title{
Some new nonlinear second-order boundary value problems on an arbitrary domain
}

\author{
Ahmed Alsaedi ${ }^{1}$, Mona Alsulami ${ }^{1}$, Ravi P. Agarwal ${ }^{1,2,3}$ and Bashir Ahmad ${ }^{1 *}$ (D)
}

*Correspondence:

bashirahmad_qau@yahoo.com ${ }^{1}$ Nonlinear Analysis and Applied Mathematics (NAAM) - Research Group, Department of Mathematics, Faculty of Science, King Abdulaziz University, Jeddah, Saudi Arabia Full list of author information is available at the end of the article
MSC: $34 \mathrm{~B} 10 ; 34 \mathrm{~B} 15$

Keywords: Ordinary differential equations; Integral; Multi-point boundary conditions; Existence; Ulam-stability

\section{Introduction}

Mathematical modeling of several real world phenomena leads to the occurrence of nonlinear boundary value problems of differential equations. In fact, the study of boundary value problems has developed into an important area of research over the years in view of their extensive applications in diverse disciplines such as fluid mechanics, mathematical physics, etc. For details, we refer the reader to the works [1-4]. In the analysis of nonlinear boundary value problems, we are primarily interested in examining the effect of nonlinearity on the solutions of the given problem. The available literature on the topic contains theoretical development of the topic as well as analytic and numerical methods for solving boundary value problems. Classical boundary conditions fail to take into account certain peculiarities of physical, chemical, or other processes happening inside the domain. This led to the birth of nonlocal conditions, which connect the boundary values of the unknown function to its values at some interior positions of the domain. For a detailed account of nonlocal nonlinear boundary value problems, for instance, see [5-19] and the references cited therein. On the other hand, integral boundary conditions appear in several applications of applied sciences such as blood flow problems, chemical engineering, thermoelasticity, underground water flow, population dynamics, etc. In particular, integral 
boundary conditions enable to consider arbitrary shaped blood vessels in fluid flow problems in contrast to assuming very long circular vessel geometry upstream the inlet section [20], which is not always justifiable. Integral boundary conditions find useful applications in blood flow problems [21, 22], thermal conduction, semiconductor, and hydrodynamic problems [23-25]. One can find some works on boundary value problems involving integral boundary conditions in a series of papers [26-33] and the references cited therein.

In this paper, we develop the existence theory for a new class of second-order boundary value problems involving nonlocal non-separated type multi-point and strip boundary conditions on an arbitrary domain. Precisely, we investigate the following problem:

$$
\left\{\begin{array}{l}
u^{\prime \prime}(t)=f(t, u(t)), \quad a<t<T, a, T \in \mathbb{R} \\
\alpha_{1} u(a)+\alpha_{2} u(T)=\alpha_{3} \int_{a}^{\xi} u(s) d s+\sum_{j=1}^{m} \gamma_{j} u\left(v_{j}\right) \\
\beta_{1} u^{\prime}(a)+\beta_{2} u^{\prime}(T)=\beta_{3} \int_{a}^{\xi} u^{\prime}(s) d s+\sum_{j=1}^{m} \rho_{j} u^{\prime}\left(v_{j}\right)
\end{array}\right.
$$

where $f:[a, T] \times \mathbb{R} \rightarrow \mathbb{R}$ is a given continuous function, $a>0, a<\xi<v_{1}<v_{2}<\cdots<$ $v_{m}<T$, and $\alpha_{i}, \beta_{i} \in \mathbb{R}(i=1,2,3), \gamma_{j}, \rho_{j} \in \mathbb{R}^{+}(j=1,2, \ldots, m)$.

The rest of the paper is organized as follows. In Sect. 2, we present the existence results for the boundary value problem (1.1), which are proved via fixed point theorems due to Schaefer, Krasnoselskii, and Leray-Schauder. The uniqueness of solutions for the given problem is established by applying contraction mapping principle. In order to illustrate the obtained results, we construct several examples. In Sect. 3, we discuss Ulam-type stability for problem (1.1). A problem analogue to (1.1) with different nonlocal data is discussed in Sect. 4. An associated boundary value problem involving integro-differential equations is studied in Sect. 5.

\section{Existence and uniqueness results}

Before presenting the main results for the nonlinear boundary value problem with nonseparated type integral multi-point boundary conditions on an arbitrary domain, we prove an auxiliary lemma. This lemma plays a key role in defining a solution for problem (1.1).

Lemma 2.1 Let $h \in C[a, T]$ and

$$
\left(\alpha_{1}+\alpha_{2}-\alpha_{3}(\xi-a)-\sum_{j=1}^{m} \gamma_{j}\right)\left(\beta_{1}+\beta_{2}-\beta_{3}(\xi-a)-\sum_{j=1}^{m} \rho_{j}\right) \neq 0 .
$$

Then an integral representation for the solution of the following linear problem:

$$
\left\{\begin{array}{l}
u^{\prime \prime}(t)=h(t), \quad a<t<T \\
\alpha_{1} u(a)+\alpha_{2} u(T)=\alpha_{3} \int_{a}^{\xi} u(s) d s+\sum_{j=1}^{m} \gamma_{j} u\left(v_{j}\right) \\
\beta_{1} u^{\prime}(a)+\beta_{2} u^{\prime}(T)=\beta_{3} \int_{a}^{\xi} u^{\prime}(s) d s+\sum_{j=1}^{m} \rho_{j} u^{\prime}\left(v_{j}\right)
\end{array}\right.
$$

is given by

$$
\begin{aligned}
u(t)= & \int_{a}^{t}(t-s) h(s) d s+\frac{1}{2 \kappa} \int_{a}^{\xi}\left[B \alpha_{3}(\xi-s)+2 \beta_{3} \eta(t)\right](\xi-s) h(s) d s \\
& -\frac{1}{\kappa} \int_{a}^{T}\left[B \alpha_{2}(T-s)+\beta_{2} \eta(t)\right] h(s) d s \\
& +\frac{1}{\kappa} \sum_{j=1}^{m} \int_{a}^{v_{j}}\left[B\left(v_{j}-s\right) \gamma_{j}+\rho_{j} \eta(t)\right] h(s) d s,
\end{aligned}
$$


where

$$
\begin{aligned}
& \kappa=B A_{1}, \quad \eta(t)=A_{1}(t-a)-A_{2}, \\
& B=\beta_{1}+\beta_{2}-\beta_{3}(\xi-a)-\sum_{j=1}^{m} \rho_{j}, \quad A_{1}=\alpha_{1}+\alpha_{2}-\alpha_{3}(\xi-a)-\sum_{j=1}^{m} \gamma_{j}, \\
& A_{2}=\alpha_{2}(T-a)-\alpha_{3} \frac{(\xi-a)^{2}}{2}-\sum_{j=1}^{m} \gamma_{j}\left(\nu_{j}-a\right) .
\end{aligned}
$$

Proof Integrating $u^{\prime \prime}(t)=h(t)$ twice from $a$ to $t$, we get

$$
u(t)=c_{1}+c_{2}(t-a)+\int_{a}^{t}(t-s) h(s) d s,
$$

where $c_{1}$ and $c_{2}$ are arbitrary real constants. Using the boundary conditions of (2.2) in (2.5), we get

$$
\begin{aligned}
& A_{1} c_{1}+A_{2} c_{2}= \alpha_{3} \int_{a}^{\xi} \frac{(\xi-s)^{2}}{2} h(s) d s-\alpha_{2} \int_{a}^{T}(T-s) h(s) d s \\
&+\sum_{j=1}^{m} \gamma_{j} \int_{a}^{v_{j}}\left(v_{j}-s\right) h(s) d s, \\
& c_{2}=\frac{1}{B}\left[-\beta_{2} \int_{a}^{T} h(s) d s+\beta_{3} \int_{a}^{\xi}(\xi-s) h(s) d s+\sum_{j=1}^{m} \rho_{j} \int_{a}^{v_{j}} h(s) d s\right] .
\end{aligned}
$$

Using (2.7) in (2.6) yields

$$
\begin{aligned}
c_{1}= & \frac{1}{\kappa}\left[B\left(\alpha_{3} \int_{a}^{\xi} \frac{(\xi-s)^{2}}{2} h(s) d s-\alpha_{2} \int_{a}^{T}(T-s) h(s) d s+\sum_{j=1}^{m} \gamma_{j} \int_{a}^{v_{j}}\left(v_{j}-s\right) h(s) d s\right)\right. \\
& \left.-A_{2}\left(-\beta_{2} \int_{a}^{T} h(s) d s+\beta_{3} \int_{a}^{\xi}(\xi-s) h(s) d s+\sum_{j=1}^{m} \rho_{j} \int_{a}^{v_{j}} h(s) d s\right)\right],
\end{aligned}
$$

where $\kappa, B$, and $A_{2}$ are given by (2.4). Substituting the values of $c_{1}$ and $c_{2}$ in (2.5) gives solution (2.3). The converse of the lemma follows by direct computation. This completes the proof.

In view of Lemma 2.1, we transform problem (1.1) into an equivalent fixed point problem

$$
u=\mathcal{G} u,
$$

where $\mathcal{G}: \mathcal{P} \rightarrow \mathcal{P}$ is defined by

$$
\begin{aligned}
(\mathcal{G} u)(t)= & \int_{a}^{t}(t-s) f(s, u(s)) d s+\frac{1}{2 \kappa} \int_{a}^{\xi}\left[B \alpha_{3}(\xi-s)+2 \beta_{3} \eta(t)\right](\xi-s) f(s, u(s)) d s \\
& -\frac{1}{\kappa} \int_{a}^{T}\left[B \alpha_{2}(T-s)+\beta_{2} \eta(t)\right] f(s, u(s)) d s \\
& +\frac{1}{\kappa} \sum_{j=1}^{m} \int_{a}^{v_{j}}\left[B\left(v_{j}-s\right) \gamma_{j}+\rho_{j} \eta(t)\right] f(s, u(s)) d s,
\end{aligned}
$$


and $\mathcal{P}$ denotes the Banach space of all continuous functions from $[a, T] \rightarrow \mathbb{R}$ endowed with the norm $\|u\|=\sup \{|u(t)|, t \in[a, T]\}$.

Notice that problem (1.1) has solutions if and only if the operator equation (2.9) has fixed points.

For the sake of computational convenience, we set

$$
\begin{aligned}
Q= & \frac{(T-a)^{2}}{2}+\left|\frac{A_{1}(T-a)-A_{2}}{\kappa}\right|\left[\left|\beta_{3}\right| \frac{(\xi-a)^{2}}{2}+\left|\beta_{2}\right|(T-a)+\sum_{j=1}^{m} \rho_{j}\left(v_{j}-a\right)\right] \\
& +\frac{1}{\left|A_{1}\right|}\left[\left|\alpha_{3}\right| \frac{(\xi-a)^{3}}{3 !}+\left|\alpha_{2}\right| \frac{(T-a)^{2}}{2}+\sum_{j=1}^{m} \gamma_{j} \frac{\left(v_{j}-a\right)^{2}}{2}\right] .
\end{aligned}
$$

\subsection{Existence results}

In this subsection, we discuss the existence of solutions for problem (1.1). Our first existence result is based on the following (Schaefer like) fixed point theorem.

Lemma 2.2 ([34]) Let $X$ be a Banach space. Assume that $\Omega: X \rightarrow X$ is a completely continuous operator and the set $Y=\{u \in X \mid u=\lambda \Omega u, 0<\lambda<1\}$ is bounded. Then $\Omega$ has a fixed point in $X$.

Theorem 2.3 Let $f:[a, T] \times \mathbb{R} \rightarrow \mathbb{R}$ be a continuous function. Assume that there exists a positive constant $L_{1}$ such that $|f(t, u(t))| \leq L_{1}$ for all $t \in[a, T], u \in \mathbb{R}$. Then problem (1.1) has at least one solution on $[a, T]$.

Proof In the first step, we show that the operator $\mathcal{G}$ defined by (2.10) is completely continuous. Observe that the continuity of $\mathcal{G}$ follows from the continuity of $f$. For a positive constant $w$, let $B_{w}=\{u \in \mathcal{P}:\|u\| \leq w\}$ be a bounded set in $\mathcal{P}$. Then, for $t \in[a, T]$, it will be shown that the operator $\mathcal{G}$ maps bounded sets into bounded sets of $\mathcal{P}$. For $u \in B_{w}, t \in[a, T]$, we have

$$
\begin{aligned}
\|(\mathcal{G} u)\|= & \sup _{t \in[a, T]}\left\{\mid \int_{a}^{t}(t-s) f(s, u(s)) d s\right. \\
& +\frac{1}{2 \kappa} \int_{a}^{\xi}\left[B \alpha_{3}(\xi-s)+2 \beta_{3} \eta(t)\right](\xi-s) f(s, u(s)) d s \\
& -\frac{1}{\kappa} \int_{a}^{T}\left[B \alpha_{2}(T-s)+\beta_{2} \eta(t)\right] f(s, u(s)) d s \\
& \left.+\frac{1}{\kappa} \sum_{j=1}^{m} \int_{a}^{v_{j}}\left[B\left(v_{j}-s\right) \gamma_{j}+\rho_{j} \eta(t)\right] f(s, u(s)) d s \mid\right\} \\
\leq & L_{1} \sup _{t \in[a, T]}\left\{\frac{(t-a)^{2}}{2}+\left|\frac{A_{1}(t-a)-A_{2} \mid}{\kappa}\right|\left[\beta_{3}\left|\frac{(\xi-a)^{2}}{2}+\right| \beta_{2} \mid(T-a)\right.\right. \\
& \left.\left.+\sum_{j=1}^{m} \rho_{j}\left(v_{j}-a\right)\right]+\frac{1}{\left|A_{1}\right|}\left[\left|\alpha_{3}\right| \frac{(\xi-a)^{3}}{3 !}+\left|\alpha_{2}\right| \frac{(T-a)^{2}}{2}+\sum_{j=1}^{m} \gamma_{j} \frac{\left(v_{j}-a\right)^{2}}{2}\right]\right\} \\
\leq & L_{1} Q,
\end{aligned}
$$

where $Q$ is defined by (2.11). 
Next we show that the operator $\mathcal{G}$ maps bounded sets into equicontinuous sets of $\mathcal{P}$. For $a<t_{1}<t_{2}<T$ and $u \in B_{w}$, we have

$$
\begin{aligned}
\left|(\mathcal{G} u)\left(t_{2}\right)-(\mathcal{G} u)\left(t_{1}\right)\right| & \\
\leq & \left|\int_{a}^{t_{1}}\left[\left(t_{2}-s\right)-\left(t_{1}-s\right)\right] f(s, u(s)) d s+\int_{t_{1}}^{t_{2}}\left(t_{2}-s\right) f(s, u(s)) d s\right| \\
& +\left|\frac{A_{1}\left(t_{2}-t_{1}\right)}{\kappa}\right|\left[\left|\beta_{3}\right| \int_{a}^{\xi}(\xi-s)|f(s, u(s))| d s+\left|\beta_{2}\right| \int_{a}^{T}|f(s, u(s))| d s\right. \\
& \left.+\sum_{j=1}^{m} \rho_{j} \int_{a}^{v_{j}}|f(s, u(s))| d s\right] \\
\leq & L_{1}\left[\left(t_{2}-t_{1}\right)\left(t_{1}-a\right)+\frac{\left(t_{2}-t_{1}\right)^{2}}{2}\right]+L_{1} \frac{1}{|B|}\left(t_{2}-t_{1}\right)\left[\left|\beta_{3}\right| \frac{(\xi-a)^{2}}{2}\right. \\
& \left.+\left|\beta_{2}\right|(T-a)+\sum_{j=1}^{m} \rho_{j}\left(v_{j}-a\right)\right] \\
\rightarrow & 0 \text { as }\left(t_{2}-t_{1}\right) \rightarrow 0,
\end{aligned}
$$

independent of $u \in B_{w}$. Therefore, by the Arzelá-Ascoli theorem, the operator $\mathcal{G}: \mathcal{P} \rightarrow \mathcal{P}$ is completely continuous.

Finally, we consider the set $V=\{u \in \mathcal{P}: u=\lambda \mathcal{G} u, 0<\lambda<1\}$ and show that $V$ is bounded. For $u \in V$ and $t \in[a, T]$, as before, we can obtain

$$
\|u\|=\sup _{t \in[a, T]}|\lambda(\mathcal{G} u)(t)| \leq L_{1} Q
$$

where $Q$ is defined by (2.11). This shows that $V$ is bounded. Hence, by Lemma 2.2, problem (1.1) has at least one solution on $[a, T]$.

We make use of Krasnoselskii's fixed point theorem [34] to establish our next result.

Lemma 2.4 (Krasnoselskii's fixed point theorem) Let $Y$ be a closed bounded, convex, and nonempty subset of a Banach space X. Let $\phi_{1}, \phi_{2}$ be the operators such that (i) $\phi_{1} y_{1}+\phi_{2} y_{2} \in$ $Y$ whenever $y_{1}, y_{2} \in Y$; (ii) $\phi_{1}$ is compact and continuous; and (iii) $\phi_{2}$ is a contraction mapping. Then there exists $y_{3} \in Y$ such that $y_{3}=\phi_{1} y_{3}+\phi_{2} y_{3}$.

Theorem 2.5 Let $f:[a, T] \times \mathbb{R} \rightarrow \mathbb{R}$ be a continuous function such that the following conditions hold:

$\left(H_{1}\right)|f(t, u)-f(t, v)| \leq \ell|u-v|, \forall t \in[a, T], \ell>0, u, v \in \mathbb{R}$.

$\left(H_{2}\right)$ There exists a function $\mu \in C\left([a, T], \mathbb{R}^{+}\right)$with $\|\mu\|=\sup _{t \in[a, T]}|\mu(t)|$ such that $|f(t, u)| \leq \mu(t), \forall(t, u) \in[a, T] \times \mathbb{R}$.

Then there exists at least one solution for problem (1.1) on $[a, T]$ if

$$
\begin{gathered}
\ell\left\{\frac{A_{1}(T-a)-A_{2}}{\kappa} \mid\left[\left|\beta_{3}\right| \frac{(\xi-a)^{2}}{2}+\left|\beta_{2}\right|(T-a)+\sum_{j=1}^{m} \rho_{j}\left(v_{j}-a\right)\right]\right. \\
\left.+\frac{1}{\left|A_{1}\right|}\left[\left|\alpha_{3}\right| \frac{(\xi-a)^{3}}{3 !}+\left|\alpha_{2}\right| \frac{(T-a)^{2}}{2}+\sum_{j=1}^{m} \gamma_{j} \frac{\left(v_{j}-a\right)^{2}}{2}\right]\right\}<1 .
\end{gathered}
$$


Proof Consider a closed ball $B_{r}=\{u \in \mathcal{P}:\|u\| \leq r\}$ with $r \geq Q\|\mu\|$, where $Q$ is given by (2.11). Introduce the operators $\mathcal{G}_{1}$ and $\mathcal{G}_{2}$ on $B_{r}$ as follows:

$$
\begin{aligned}
\left(\mathcal{G}_{1} u\right)(t)= & \int_{a}^{t}(t-s) f(s, u(s)) d s, \\
\left(\mathcal{G}_{2} u\right)(t)= & \frac{1}{2 \kappa} \int_{a}^{\xi}\left[B \alpha_{3}(\xi-s)+2 \beta_{3} \eta(t)\right](\xi-s) f(s, u(s)) d s \\
& -\frac{1}{\kappa} \int_{a}^{T}\left[B \alpha_{2}(T-s)+\beta_{2} \eta(t)\right] f(s, u(s)) d s \\
& +\frac{1}{\kappa} \sum_{j=1}^{m} \int_{a}^{v_{j}}\left[B\left(v_{j}-s\right) \gamma_{j}+\rho_{j} \eta(t)\right] f(s, u(s)) d s .
\end{aligned}
$$

Notice that $\mathcal{G}=\mathcal{G}_{1}+\mathcal{G}_{2}$. For $u, v \in B_{r}$, we have

$$
\begin{aligned}
\left\|\mathcal{G}_{1} u+\mathcal{G}_{2} v\right\|= & \sup _{t \in[a, T]}\left\{\mid \int_{a}^{t}(t-s) f(s, u(s)) d s\right. \\
& +\frac{1}{2 \kappa} \int_{a}^{\xi}\left[B \alpha_{3}(\xi-s)+2 \beta_{3} \eta(t)\right](\xi-s) f(s, v(s)) d s \\
& -\frac{1}{\kappa} \int_{a}^{T}\left[B \alpha_{2}(T-s)+\beta_{2} \eta(t)\right] f(s, v(s)) d s \\
& \left.+\frac{1}{\kappa} \sum_{j=1}^{m} \int_{a}^{v_{j}}\left[B\left(v_{j}-s\right) \gamma_{j}+\rho_{j} \eta(t)\right] f(s, v(s)) d s \mid\right\} \\
\leq & \|\mu\| \sup _{t \in[a, T]}\left\{\frac{(t-a)^{2}}{2}+\left|\frac{A_{1}(t-a)-A_{2} \mid}{\kappa}\right|\left[\left|\beta_{3}\right| \frac{(\xi-a)^{2}}{2}\right.\right. \\
& \left.+\left|\beta_{2}\right|(T-a)+\sum_{j=1}^{m} \rho_{j}\left(v_{j}-a\right)\right]+\frac{1}{\left|A_{1}\right|}\left[\left|\alpha_{3}\right| \frac{(\xi-a)^{3}}{3 !}+\left|\alpha_{2}\right| \frac{(T-a)^{2}}{2}\right. \\
& \left.\left.+\sum_{j=1}^{m} \gamma_{j} \frac{\left(v_{j}-a\right)^{2}}{2}\right]\right\} \\
\leq & \|\mu\| Q \leq r .
\end{aligned}
$$

Thus, $\mathcal{G}_{1} u+\mathcal{G}_{2} v \in B_{r}$, which verifies assumption (i) in Lemma 2.4. Using assumption $\left(H_{1}\right)$, we obtain

$$
\begin{aligned}
\left\|\mathcal{G}_{2} u-\mathcal{G}_{2} v\right\|= & \sup _{t \in[a, T]} \mid \frac{1}{2 \kappa} \int_{a}^{\xi}\left[B \alpha_{3}(\xi-s)+2 \beta_{3} \eta(t)\right](\xi-s)(f(s, u(s))-f(s, v(s))) d s \\
& -\frac{1}{\kappa} \int_{a}^{T}\left[B \alpha_{2}(T-s)+\beta_{2} \eta(t)\right](f(s, u(s))-f(s, v(s))) d s \\
& +\frac{1}{\kappa} \sum_{j=1}^{m} \int_{a}^{v_{j}}\left[B\left(v_{j}-s\right) \gamma_{j}+\rho_{j} \eta(t)\right](f(s, u(s))-f(s, v(s))) d s \mid
\end{aligned}
$$




$$
\begin{aligned}
\leq & \ell\left|\frac{A_{1}(T-a)-A_{2}}{\kappa}\right|\left[\left|\beta_{3}\right| \frac{(\xi-a)^{2}}{2}+\left|\beta_{2}\right|(T-a)+\sum_{j=1}^{m} \rho_{j}\left(v_{j}-a\right)\right] \\
& \left.+\frac{1}{\left|A_{1}\right|}\left[\left|\alpha_{3}\right| \frac{(\xi-a)^{3}}{3 !}+\left|\alpha_{2}\right| \frac{(T-a)^{2}}{2}+\sum_{j=1}^{m} \gamma_{j} \frac{\left(v_{j}-a\right)^{2}}{2}\right]\right\}\|u-v\|,
\end{aligned}
$$

which, in view of condition (2.12), shows that $\mathcal{G}_{2}$ is a contraction.

Next, we show that $\mathcal{G}_{1}$ is compact and continuous. Notice that the continuity of $f$ implies that the operator $\mathcal{G}_{1}$ is continuous. Also, $\mathcal{G}_{1}$ is uniformly bounded on $B_{r}$ as

$$
\left\|\mathcal{G}_{1} u\right\| \leq\|\mu\| \frac{(T-a)^{2}}{2} .
$$

Let us fix $\sup _{(t, u) \in[a, T] \times B_{r}}|f(t, u)|=\bar{f}$, and take $a<t_{1}<t_{2}<T$. Then

$$
\begin{aligned}
\left|\left(\mathcal{G}_{1} u\right)\left(t_{2}\right)-\left(\mathcal{G}_{1} u\right)\left(t_{1}\right)\right| & =\left|\int_{a}^{t_{1}}\left(\left(t_{2}-s\right)-\left(t_{1}-s\right)\right) f(s, u(s)) d s+\int_{t_{1}}^{t_{2}}\left(t_{2}-s\right) f(s, u(s)) d s\right| \\
& \leq \bar{f}\left|\left(t_{2}-t_{1}\right)\left(t_{1}-a\right)+\frac{\left(t_{2}-t_{1}\right)^{2}}{2}\right| \rightarrow 0 \quad \text { as }\left(t_{2}-t_{1}\right) \rightarrow 0,
\end{aligned}
$$

independently of $u \in B_{r}$. This implies that $\mathcal{G}_{1}$ is relatively compact on $B_{r}$. Hence, it follows by the Arzelá-Ascoli theorem that the operator $\mathcal{G}_{1}$ is compact on $B_{r}$. Thus all the assumptions of Lemma 2.4 are satisfied. In consequence, by the conclusion of Lemma 2.4, problem (1.1) has at least one solution on $[a, T]$.

Remark 2.6 If we interchange the role of the operators $\mathcal{G}_{1}$ and $\mathcal{G}_{2}$ in the previous theorem, then condition (2.12) takes the form $\ell \frac{(T-a)^{2}}{2}<1$.

In the next result, we make use of Leray-Schauder nonlinear alternative for singlevalued maps to develop existence criteria for solutions of problem (1.1).

Lemma 2.7 (Leray-Schauder alternative [35]) Let $C$ be a closed and convex subset of a Banach space $E$ and $U$ be an open subset of $C$ with $0 \in U$. Suppose that $\mathcal{V}: \bar{U} \rightarrow C$ is a continuous, compact (that is, $\mathcal{V}(\bar{U})$ is a relatively compact subset of $C$ ) map. Then either (i) $\mathcal{V}$ has a fixed point in $\bar{U}$, or (ii) there are $u \in \partial U$ (the boundary of $U$ in $C$ ) and $\lambda \in(0,1)$ with $u=\lambda \mathcal{V}(u)$.

Theorem 2.8 Let $f:[a, T] \times \mathbb{R} \rightarrow \mathbb{R}$ be a continuous function. Assume that

$\left(H_{3}\right)$ There exist a function $p \in C\left([a, T], \mathbb{R}^{+}\right)$and a nondecreasing function $\Psi: \mathbb{R}^{+} \rightarrow \mathbb{R}^{+}$ such that $|f(t, u)| \leq p(t) \Psi(\|u\|), \forall(t, u) \in[a, T] \times \mathbb{R}$;

$\left(H_{4}\right)$ There exists a constant $M>0$ such that

$$
\frac{M}{\|p\| \Psi(M) Q}>1
$$

where $Q$ is given by (2.11).

Then problem (1.1) has at least one solution on $[a, T]$. 
Proof We complete the proof in several steps. In the first step we show that the operator $\mathcal{G}: \mathcal{P} \rightarrow \mathcal{P}$ defined by (2.10) maps bounded sets into bounded sets in $\mathcal{P}$. For the positive number $r$, let $B_{r}=\{u \in \mathcal{P}:\|u\| \leq r\}$ be a bounded set in $\mathcal{P}$. Then

$$
\begin{aligned}
\|(\mathcal{G} u)\|= & \sup _{t \in[a, T]} \mid \int_{a}^{t}(t-s) f(s, u(s)) d s \\
& +\frac{1}{2 \kappa} \int_{a}^{\xi}\left[B \alpha_{3}(\xi-s)+2 \beta_{3} \eta(t)\right](\xi-s) f(s, u(s)) d s \\
& -\frac{1}{\kappa} \int_{a}^{T}\left[B \alpha_{2}(T-s)+\beta_{2} \eta(t)\right] f(s, u(s)) d s \\
& +\frac{1}{\kappa} \sum_{j=1}^{m} \int_{a}^{v_{j}}\left[B\left(v_{j}-s\right) \gamma_{j}+\rho_{j} \eta(t)\right] f(s, u(s)) d s \mid \\
\leq & \|p\| \Psi(\|u\|) \sup _{t \in[a, T]}\left\{\frac{(t-a)^{2}}{2}+\left|\frac{A_{1}(t-a)-A_{2} \mid}{\kappa}\right|\left[\beta_{3} \mid \frac{(\xi-a)^{2}}{2}\right.\right. \\
& \left.+\left|\beta_{2}\right|(T-a)+\sum_{j=1}^{m} \rho_{j}\left(v_{j}-a\right)\right]+\frac{1}{\left|A_{1}\right|}\left[\left|\alpha_{3}\right| \frac{(\xi-a)^{3}}{3 !}+\left|\alpha_{2}\right| \frac{(T-a)^{2}}{2}\right. \\
& \left.\left.+\sum_{j=1}^{m} \gamma_{j} \frac{\left(v_{j}-a\right)^{2}}{2}\right]\right\} \\
\leq & \|p\| \Psi(\|u\|) Q \leq\|p\| \Psi(r) Q .
\end{aligned}
$$

Next we show that $\mathcal{G}$ maps bounded sets into equicontinuous sets of $\mathcal{P}$. Observe that the continuity of $\mathcal{G}$ follows from the continuity of $f$. As shown in the proof of Theorem 2.3, the operator $\mathcal{G}: \mathcal{P} \rightarrow \mathcal{P}$ is completely continuous. The result will follow from the LeraySchauder nonlinear alternative (Lemma 2.7) once we establish the boundedness of the set of all solutions to the equation $u=\lambda \mathcal{G} u$ for $\lambda \in[0,1]$. Let $u$ be a solution. Then, for $t \in[a, T]$, computing as in the first step, we have

$$
|u(t)|=|\lambda(\mathcal{G} u)(t)| \leq\|p\| \Psi(r) Q
$$

which, on taking the norm for $t \in[a, T]$, yields

$$
\frac{\|u\|}{\|p\| \Psi(r) Q} \leq 1
$$

In view of $\left(H_{4}\right)$, there exists $M$ such that $\|u\| \neq M$. Let us set

$$
U=\{u \in \mathcal{P}:\|u\|<M\}
$$

Note that the operator $\mathcal{G}: \bar{U} \rightarrow \mathcal{P}$ is continuous and completely continuous. From the choice of $U$, there is no $u \in \partial U$ such that $u=\lambda \mathcal{G}(u)$ for some $\lambda \in(0,1)$. Consequently, it follows by Lemma 2.7 that the operator $\mathcal{G}$ has a fixed point $u \in \bar{U}$ which is a solution of problem (1.1). This completes the proof. 


\subsection{Uniqueness of solutions}

Here we establish the uniqueness of solutions for problem (1.1) by means of Banach's contraction mapping principle.

Theorem 2.9 Assume that $f:[a, T] \times \mathbb{R} \rightarrow \mathbb{R}$ is a continuous function satisfying the Lipschitz condition $\left(H_{1}\right)$. Then there exists a unique solution for problem (1.1) on $[a, T]$ if $\ell<1 / Q$, where $Q$ is given by (2.11).

Proof Let us fix $\sup _{t \in[a, T]}|f(t, 0)|=N, w \geq \frac{Q N}{1-\ell Q}$ and show that $\mathcal{G} B_{w} \subset B_{w}$, where $B_{w}=$ $\{u \in \mathcal{P}:\|u\| \leq w\}$. For any $u \in B_{w}, t \in[a, T]$, we find that

$$
\begin{aligned}
|f(s, u(s))| & =|f(s, u(s))-f(s, 0)+f(s, 0)| \leq|f(s, u(s))-f(s, 0)|+|f(s, 0)| \\
& \leq \ell\|u\|+N \leq \ell w+N .
\end{aligned}
$$

Then, for $u \in B_{w}$, we obtain

$$
\begin{aligned}
\|(\mathcal{G} u)\|= & \sup _{t \in[a, T]} \mid \int_{a}^{t}(t-s) f(s, u(s)) d s \\
& +\frac{1}{2 \kappa} \int_{a}^{\xi}\left[B \alpha_{3}(\xi-s)+2 \beta_{3} \eta(t)\right](\xi-s) f(s, u(s)) d s \\
& -\frac{1}{\kappa} \int_{a}^{T}\left[B \alpha_{2}(T-s)+\beta_{2} \eta(t)\right] f(s, u(s)) d s \\
& +\frac{1}{\kappa} \sum_{j=1}^{m} \int_{a}^{v_{j}}\left[B\left(v_{j}-s\right) \gamma_{j}+\rho_{j} \eta(t)\right] f(s, u(s)) d s \mid \\
\leq & (\ell w+N) \sup _{t \in[a, T]}\left\{\frac{(t-a)^{2}}{2}+\left|\frac{A_{1}(t-a)-A_{2} \mid}{\kappa}\right|\left[\left|\beta_{3}\right| \frac{(\xi-a)^{2}}{2}\right.\right. \\
& \left.+\left|\beta_{2}\right|(T-a)+\sum_{j=1}^{m} \rho_{j}\left(v_{j}-a\right)\right]+\frac{1}{\left|A_{1}\right|}\left[\left|\alpha_{3}\right| \frac{(\xi-a)^{3}}{3 !}+\left|\alpha_{2}\right| \frac{(T-a)^{2}}{2}\right. \\
& \left.\left.+\sum_{j=1}^{m} \gamma_{j} \frac{\left(v_{j}-a\right)^{2}}{2}\right]\right\} \\
\leq & (\ell w+N) Q \leq w,
\end{aligned}
$$

where $Q$ is given by (2.11). This shows that $\mathcal{G} B_{w} \subset B_{w}$.

Next we show that the operator $\mathcal{G}$ is a contraction. For $u, v \in \mathcal{P}$, we obtain

$$
\begin{aligned}
\|\mathcal{G} u-\mathcal{G} v\|= & \sup _{t \in[0, T]}|\mathcal{G} u(t)-\mathcal{G} v(t)| \\
\leq & \sup _{t \in[a, T]} \mid \int_{a}^{t}(t-s)(f(s, u(s))-f(s, v(s))) d s \\
& +\frac{1}{2 \kappa} \int_{a}^{\xi}\left[B \alpha_{3}(\xi-s)+2 \beta_{3} \eta(t)\right](\xi-s)(f(s, u(s))-f(s, v(s))) d s \\
& -\frac{1}{\kappa} \int_{a}^{T}\left[B \alpha_{2}(T-s)+\beta_{2} \eta(t)\right](f(s, u(s))-f(s, v(s))) d s
\end{aligned}
$$




$$
\begin{aligned}
& +\frac{1}{\kappa} \sum_{j=1}^{m} \int_{a}^{v_{j}}\left[B\left(v_{j}-s\right) \gamma_{j}+\rho_{j} \eta(t)\right](f(s, u(s))-f(s, v(s))) d s \mid \\
\leq & \ell\|u-v\|\left\{\frac{(T-a)^{2}}{2}+\frac{\left|A_{1}(T-a)-A_{2}\right|}{|\kappa|}\left[\left|\beta_{3}\right| \frac{(\xi-a)^{2}}{2}\right.\right. \\
& \left.+\left|\beta_{2}\right|(T-a)+\sum_{j=1}^{m} \rho_{j}\left(v_{j}-a\right)\right]+\frac{1}{\left|A_{1}\right|}\left[\left|\alpha_{3}\right| \frac{(\xi-a)^{3}}{3 !}+\left|\alpha_{2}\right| \frac{(T-a)^{2}}{2}\right. \\
& \left.\left.+\sum_{j=1}^{m} \gamma_{j} \frac{\left(v_{j}-a\right)^{2}}{2}\right]\right\} \\
\leq & \ell Q\|u-v\|,
\end{aligned}
$$

where we have used (2.11). By the given assumption $\ell<1 / Q$, it follows that the operator $\mathcal{G}: \mathcal{P} \rightarrow \mathcal{P}$ is a contraction. Thus, by Banach's contraction mapping principle, we deduce that the operator $\mathcal{G}$ has a fixed point, which corresponds to a unique solution of problem (1.1) on $[a, T]$.

\subsection{Examples}

In this subsection, we illustrate the results obtained in the last two subsections with the aid of examples.

Example 2.10 Consider the following non-separated multi-point boundary value problem:

$$
\left\{\begin{array}{l}
u^{\prime \prime}(t)=\frac{1}{\sqrt{t+8}} \tan ^{-1} u(t)+\sin t, \quad 1<t<2, \\
\alpha_{1} u(1)+\alpha_{2} u(2)=\alpha_{3} \int_{a}^{\xi} u(s) d s+\sum_{j=1}^{4} \gamma_{j} u\left(v_{j}\right), \\
\beta_{1} u^{\prime}(1)+\beta_{2} u^{\prime}(2)=\beta_{3} \int_{a}^{\xi} u^{\prime}(s) d s+\sum_{j=1}^{4} \rho_{j} u^{\prime}\left(v_{j}\right),
\end{array}\right.
$$

where $a=1, T=2, m=4, \alpha_{1}=1, \alpha_{2}=1 / 2, \alpha_{3}=1, \beta_{1}=1, \beta_{2}=1, \beta_{3}=1, \gamma_{1}=1 / 4, \gamma_{2}=5 / 12$, $\gamma_{3}=7 / 12, \gamma_{4}=9 / 12, \rho_{1}=2 / 5, \rho_{2}=11 / 15, \rho_{3}=16 / 15, \rho_{4}=7 / 5, \xi=5 / 4, v_{1}=9 / 7, v_{2}=79 / 56$, $\nu_{3}=43 / 28, v_{4}=93 / 56$. Clearly, $|f(t, u)| \leq \frac{\pi}{2 \sqrt{t+8}}+1$, and $|f(t, u)-f(t, v)| \leq \ell|u-v|$, with $\ell=1 / 3$. Using the given values, we find that $\left|A_{1}\right|=0.75 \neq 0,\left|A_{2}\right|=0.581845,|B|=1.85 \neq 0$, $|\kappa|=1.3875\left(\kappa, B, A_{1}\right.$, and $A_{2}$ are given by (2.4)) and $Q=1.583835$ ( $Q$ is given by (2.11)). Also we have

$$
\begin{aligned}
& \ell\left\{\frac{\left|A_{1}(T-a)-A_{2}\right|\left[\left|\beta_{3}\right| \frac{(\xi-a)^{2}}{2}+\left|\beta_{2}\right|(T-a)+\sum_{j=1}^{m} \rho_{j}\left(v_{j}-a\right)\right]}{\kappa}+\frac{1}{\left|A_{1}\right|}\left[\left|\alpha_{3}\right| \frac{(\xi-a)^{3}}{3 !}+\left|\alpha_{2}\right| \frac{(T-a)^{2}}{2}+\sum_{j=1}^{m} \gamma_{j} \frac{\left(v_{j}-a\right)^{2}}{2}\right]\right\} \approx 0.361278<1,
\end{aligned}
$$

that is, condition (2.12) is satisfied. Thus all the conditions of Theorem 2.5 are satisfied; and consequently the conclusion of Theorem 2.5 applies to problem (2.14). On the other hand, as $\ell Q \approx 0.527945<1$, it follows by Theorem 2.9 that there exists a unique solution for problem (2.14) on [1,2]. 
Example 2.11 Consider the nonlinear second-order ordinary differential equation

$$
u^{\prime \prime}(t)=\frac{7}{3 \sqrt{t+48}}\left(\frac{1}{5} \frac{|u|}{|u|+1}+\sin u+\frac{5}{2}\right)
$$

supplemented with the boundary conditions of problem (2.14). Evidently, $|f(t, u)| \leq$ $p(t) \Psi(\|u\|)$ with $\Psi(\|u\|)=\|u\|+\frac{27}{10}, p(t)=\frac{7}{3 \sqrt{t+48}}$ and $\|p\|=\frac{1}{3}$. Condition (2.13) implies that $M>1.906557$. Thus we deduce by Theorem 2.8 that problem (2.14) has at least one solution on $[1,2]$.

\section{Ulam stability}

In this section, we develop the criteria for Ulam stability of problem (1.1) by means of its equivalent integral equation

$$
\begin{aligned}
v(t)= & \int_{a}^{t}(t-s) f(s, v(s)) d s+\frac{1}{2 \kappa} \int_{a}^{\xi}\left[B \alpha_{3}(\xi-s)+2 \beta_{3} \eta(t)\right](\xi-s) f(s, v(s)) d s \\
& -\frac{1}{\kappa} \int_{a}^{T}\left[B \alpha_{2}(T-s)+\beta_{2} \eta(t)\right] f(s, v(s)) d s \\
& +\frac{1}{\kappa} \sum_{j=1}^{m} \int_{a}^{v_{j}}\left[B\left(v_{j}-s\right) \gamma_{j}+\rho_{j} \eta(t)\right] f(s, v(s)) d s,
\end{aligned}
$$

where $v \in \mathcal{P}$ and $f:[a, T] \times \mathbb{R} \rightarrow \mathbb{R}$ is a continuous function. Next, we define a continuous nonlinear operator $\Phi: \mathcal{P} \rightarrow \mathcal{P}$ as

$$
\Phi v(t)=D^{2} v(t)-f(t, v(t))
$$

Definition 3.1 Problem (1.1) is said to be Ulam-Hyers stable if there exists a real number $c>0$ such that, for each $\epsilon>0$ and for each solution $v \in \mathcal{P}$,

$$
\|\Phi v\| \leq \epsilon, \quad t \in[a, T]
$$

there exists a solution $u \in \mathcal{P}$ of (1.1) satisfying the inequality

$$
\|u-v\| \leq c \epsilon_{1}, \quad t \in[a, T]
$$

where $\epsilon_{1}$ is a positive real number depending on $\epsilon$.

Definition 3.2 Problem (1.1) is generalized Ulam-Hyers stable if there exists $\varrho \in$ $C\left(\mathbb{R}^{+}, \mathbb{R}^{+}\right)$such that, for each solution $v \in \mathcal{P}$ of (1.1), there exists a solution $u \in \mathcal{P}$ of (1.1) with

$$
|u(t)-v(t)| \leq \varrho(\epsilon), \quad t \in[a, T]
$$

Definition 3.3 Problem (1.1) is Ulam-Hyers-Rassias stable with respect to $\phi \in$ $C\left([a, T], \mathbb{R}^{+}\right)$if there exists a real number $c>0$ such that, for each $\epsilon>0$ and for each solution $v \in \mathcal{P}$ of (1.1),

$$
|\Phi v(t)| \leq \epsilon \phi(t), \quad t \in[a, T],
$$


we can find a solution $u \in \mathcal{P}$ of (1.1) satisfying the inequality

$$
|u(t)-v(t)| \leq c \epsilon_{1} \phi(t), \quad t \in[a, T],
$$

where $\epsilon_{1}$ is a positive real number depending on $\epsilon$.

Theorem 3.4 Assume that conditions $\left(H_{1}\right)$ and (2.12) hold. Then problem (1.1) satisfies both Ulam-Hyers and generalized Ulam-Hyers stability criteria.

Proof We know that $u \in \mathcal{P}$ is a unique solution of (1.1) (see Theorem 2.9). Let $v \in \mathcal{P}$ be another solution of (1.1) satisfying (3.2). Observe that the operators $\Phi$ and $\mathcal{G}-I$ are equivalent for every solution $v \in \mathcal{P}$ (given by (3.1)) of (1.1). Therefore, by the fixed point property of the operator $\mathcal{G}$ (given by (2.10)) together with (2.9) and (3.2), we have

$$
\begin{aligned}
|v(t)-u(t)| & =|v(t)-\mathcal{G} v(t)+\mathcal{G} v(t)-\mathcal{G} u(t)| \\
& \leq|\mathcal{G} u(t)-\mathcal{G} v(t)|+|\mathcal{G} v(t)-v(t)| \\
& \leq \ell Q_{1}\|u-v\|+\epsilon,
\end{aligned}
$$

where $\epsilon>0$ and $\ell Q_{1}<1$ is given by (2.12). Note that $Q_{1}$ denotes the coefficient of $\ell$ in (2.12). Taking the norm of (3.4) for $t \in[a, T]$ and solving for $\|u-v\|$, we obtain

$$
\|u-v\| \leq \frac{\epsilon}{1-\ell Q_{1}}
$$

If we let $\epsilon_{1}=\frac{\epsilon}{1-\ell Q_{1}}$ and $c=1$, then the Ulam-Hyers stability condition is satisfied. More generally, defining $\varrho(\epsilon)=\frac{\epsilon}{1-\ell Q_{1}}$, the generalized Ulam-Hyers stability condition is also satisfied. This completes the proof.

Theorem 3.5 Assume that conditions $\left(H_{1}\right)$ and (2.12) hold and there exists a function $\phi \in C\left([a, T], \mathbb{R}^{+}\right)$satisfying condition (3.3). Then problem (1.1) is Ulam-Hyers-Rassias stable with respect to $\phi$.

Proof Following the arguments employed in the proof of Theorem 3.4, we can obtain that

$$
\|u-v\| \leq \epsilon_{1} \phi(t)
$$

with $\epsilon_{1}=\frac{\epsilon}{1-\ell Q_{1}}$. This completes proof.

\section{An analogue problem with different nonlocal data}

This section is devoted to the study of a second-order boundary value problem involving different nonlocal data given by

$$
\left\{\begin{array}{l}
u^{\prime \prime}(t)=f(t, u(t)), \quad a<t<T, a, T \in \mathbb{R}, \\
\alpha_{1} u(a)+\alpha_{2} u(T)=\alpha_{3} \int_{a}^{\xi} u(s) d s+\sum_{j=1}^{m} \gamma_{j} u\left(v_{j}\right), \\
\beta_{1} u^{\prime}(a)+\beta_{2} u^{\prime}(T)=\beta_{3} \int_{a}^{\zeta} u^{\prime}(s) d s+\sum_{j=1}^{m} \rho_{j} u^{\prime}\left(\widehat{v}_{j}\right)
\end{array}\right.
$$


where $f:[a, T] \times \mathbb{R} \rightarrow \mathbb{R}$ is a given continuous function, $a<\xi<\zeta<\nu_{1}<\widehat{\nu_{1}}<\nu_{2}<\widehat{\nu_{2}}<\cdots<$ $v_{m}<\widehat{v_{m}}<T$, and $\alpha_{i}, \beta_{i} \in \mathbb{R}(i=1,2,3), \gamma_{j}, \rho_{j} \in \mathbb{R}^{+}(j=1,2, \ldots, m)$. The fixed point problem associated with problem (4.1) is $u=\widehat{\mathcal{G}} u$, where $\widehat{\mathcal{G}}: \mathcal{P} \rightarrow \mathcal{P}$ is defined by

$$
\begin{aligned}
(\widehat{\mathcal{G}} u)(t)= & \int_{a}^{t}(t-s) f(s, u(s)) d s-\frac{1}{\varepsilon} \int_{a}^{T}\left[J \alpha_{2}(T-s)+\beta_{2} F(t)\right] f(s, u(s)) d s \\
& +\frac{F(t)}{\varepsilon}\left[\beta_{3} \int_{a}^{\zeta}(\zeta-s)+\sum_{j=1}^{m} \rho_{j} \int_{a}^{\widehat{v}_{j}}\right] f(s, u(s)) d s \\
& +\frac{1}{F_{1}}\left[\alpha_{3} \int_{a}^{\xi} \frac{(\xi-s)^{2}}{2}+\sum_{j=1}^{m} \gamma_{j} \int_{a}^{v_{j}}\left(v_{j}-s\right)\right] f(s, u(s)) d s,
\end{aligned}
$$

$\varepsilon=J F_{1}, F(t)=F_{1}(t-a)-F_{2}, J=\beta_{1}+\beta_{2}-\beta_{3}(\zeta-a)-\sum_{j=1}^{m} \rho_{j} \neq 0, F_{1}=\alpha_{1}+\alpha_{2}-\alpha_{3}(\xi-a)-$ $\sum_{j=1}^{m} \gamma_{j} \neq 0, F_{2}=\alpha_{2}(T-a)-\alpha_{3} \frac{(\xi-a)^{2}}{2}-\sum_{j=1}^{m} \gamma_{j}\left(v_{j}-a\right)$.

Let us set

$$
\begin{aligned}
\widehat{Q}= & \frac{(T-a)^{2}}{2}+\left|\frac{F_{1}(T-a)-F_{2} \mid}{\varepsilon}\right|\left[\left|\beta_{3}\right| \frac{(\zeta-a)^{2}}{2}+\left|\beta_{2}\right|(T-a)+\sum_{j=1}^{m} \rho_{j}\left(\widehat{v}_{j}-a\right)\right] \\
& +\frac{1}{\left|F_{1}\right|}\left[\left|\alpha_{3}\right| \frac{(\xi-a)^{3}}{3 !}+\left|\alpha_{2}\right| \frac{(T-a)^{2}}{2}+\sum_{j=1}^{m} \gamma_{j} \frac{\left(v_{j}-a\right)^{2}}{2}\right] .
\end{aligned}
$$

Employing the strategy used in Sect. 3, we can obtain the existence results for problem (4.1) with the aid of the operator $\widehat{\mathcal{G}}: \mathcal{P} \rightarrow \mathcal{P}$ and the constant $\widehat{Q}$ respectively defined by (4.2) and (4.3).

\section{An integro-differential problem with non-separated type strip multi-point boundary conditions}

In this section, we consider the following second-order integro-differential boundary value problem:

$$
\left\{\begin{array}{l}
u^{\prime \prime}(t)=\lambda_{1} f(t, u(t))+\lambda_{2} \int_{a}^{t} g(s, u(s)) d s, \quad a<t<T, a, T, \lambda_{1}, \lambda_{2} \in \mathbb{R} \\
\alpha_{1} u(a)+\alpha_{2} u(T)=\alpha_{3} \int_{a}^{\xi} u(s) d s+\sum_{j=1}^{m} \gamma_{j} u\left(v_{j}\right) \\
\beta_{1} u^{\prime}(a)+\beta_{2} u^{\prime}(T)=\beta_{3} \int_{a}^{\xi} u^{\prime}(s) d s+\sum_{j=1}^{m} \rho_{j} u^{\prime}\left(v_{j}\right)
\end{array}\right.
$$

where $f:[a, T] \times \mathbb{R} \rightarrow \mathbb{R}, g:[a, T] \times \mathbb{R} \rightarrow \mathbb{R}$ are given continuous functions, $a>0, a<\xi<$ $v_{1}<v_{2}<\cdots<v_{m}<T$, and $\alpha_{i}, \beta_{i} \in \mathbb{R}(i=1,2,3), \gamma_{j}, \rho_{j} \in \mathbb{R}^{+}(j=1,2, \ldots, m)$.

The fixed point problem associated with (5.1) is $u=\overline{\mathcal{G}} u$, where $\overline{\mathcal{G}}: \mathcal{P} \rightarrow \mathcal{P}$ is defined by

$$
\begin{aligned}
(\overline{\mathcal{G}} u)(t)= & \int_{a}^{t}(t-s)\left(\lambda_{1} f(s, u(s))+\lambda_{2} \int_{a}^{s} g(p, u(p)) d p\right) d s \\
& +\frac{1}{2 \kappa} \int_{a}^{\xi}\left\{\left(B \alpha_{3}(\xi-s)+2 \beta_{3} \eta(t)\right)(\xi-s)\right. \\
& \left.\times\left(\lambda_{1} f(s, u(s))+\lambda_{2} \int_{a}^{s} g(p, u(p)) d p\right)\right\} d s \\
& -\frac{1}{\kappa} \int_{a}^{T}\left\{\left(B \alpha_{2}(T-s)+\beta_{2} \eta(t)\right)\left(\lambda_{1} f(s, u(s))+\lambda_{2} \int_{a}^{s} g(p, u(p)) d p\right)\right\} d s
\end{aligned}
$$




$$
\begin{aligned}
& +\frac{1}{\kappa} \sum_{j=1}^{m} \int_{a}^{v_{j}}\left\{\left(B\left(v_{j}-s\right) \gamma_{j}+\rho_{j} \eta(t)\right)\right. \\
& \left.\times\left(\lambda_{1} f(s, u(s))+\lambda_{2} \int_{a}^{s} g(p, u(p)) d p\right)\right\} d s,
\end{aligned}
$$

where $\kappa$, and $\eta(t)$ are given by (2.4). Now, we set

$$
\bar{Q}=\lambda_{1} Q+\lambda_{2} \bar{Q}_{1}
$$

where $Q$ is defined by (2.11) and

$$
\begin{aligned}
\bar{Q}_{1}= & \frac{(T-a)^{3}}{3 !}+\left|\frac{A_{1}(T-a)-A_{2}}{\kappa}\right|\left[\left|\beta_{3}\right| \frac{(\xi-a)^{3}}{3 !}+\left|\beta_{2}\right| \frac{(T-a)^{2}}{2}\right. \\
& \left.+\sum_{j=1}^{m} \rho_{j} \frac{\left(v_{j}-a\right)^{2}}{2}\right]+\frac{1}{\left|A_{1}\right|}\left[\left|\alpha_{3}\right| \frac{(\xi-a)^{4}}{4 !}+\left|\alpha_{2}\right| \frac{(T-a)^{3}}{3 !}+\sum_{j=1}^{m} \gamma_{j} \frac{\left(v_{j}-a\right)^{3}}{3 !}\right] .
\end{aligned}
$$

In the following result, we prove the uniqueness of solutions for problem (5.1) by means of Banach's contraction mapping principle. For that we need the following assumption:

$\left(H_{5}\right)|g(t, u)-g(t, v)| \leq \ell_{1}|u-v|, \forall t \in[a, T], \ell_{1}>0, u, v \in \mathbb{R}$.

Theorem 5.1 Let $f, g:[a, T] \times \mathbb{R} \rightarrow \mathbb{R}$ be continuous functions satisfying conditions $\left(H_{1}\right)$ and $\left(H_{5}\right)$, respectively. Then the boundary value problem (5.1) has a unique solution on $[a, T]$ if

$$
\ell \lambda_{1} Q+\ell_{1} \lambda_{2} \bar{Q}_{1}<1
$$

where $Q$ and $\bar{Q}_{1}$ are respectively given by (2.11) and (5.4).

Proof In the first step, we define $B_{\bar{w}}=\{u \in \mathcal{P}:\|u\| \leq \bar{w}\}$, where $\sup _{t \in[a, T]}|f(t, 0)|=N$, $\sup _{t \in[a, T]}|g(t, 0)|=N_{1}, \bar{w} \geq \frac{\lambda_{1} N Q+\lambda_{2} N_{1} \bar{Q}_{1}}{1-\ell \lambda_{1} Q-\ell_{1} \lambda_{2} \bar{Q}_{1}}$, and show that $\overline{\mathcal{G}} B_{\bar{w}} \subset B_{\bar{w}}$ (the operator $\overline{\mathcal{G}}$ is defined by (5.2)). Then, for any $u \in B_{\bar{w}}, t \in[a, T]$, we have

$$
\begin{aligned}
|f(s, u(s))| & =|f(s, u(s))-f(s, 0)+f(s, 0)| \leq|f(s, u(s))-f(s, 0)|+|f(s, 0)| \\
& \leq \ell\|u\|+N \leq \ell \bar{w}+N .
\end{aligned}
$$

Similarly, one can find that $|g(s, u(s))| \leq \ell_{1} \bar{w}+N_{1}$. Then, for $u \in B_{\bar{w}}$, we obtain

$$
\begin{aligned}
\|(\overline{\mathcal{G}} u)\|= & \sup _{t \in[a, T]}\left\{\mid \int_{a}^{t}(t-s)\left(\lambda_{1} f(s, u(s))+\lambda_{2} \int_{a}^{s} g(p, u(p)) d p\right) d s\right. \\
& +\frac{1}{2 \kappa} \int_{a}^{\xi}\left\{( B \alpha _ { 3 } ( \xi - s ) + 2 \beta _ { 3 } \eta ( t ) ) ( \xi - s ) \left[\lambda_{1} f(s, u(s))\right.\right. \\
& \left.\left.+\lambda_{2} \int_{a}^{s} g(p, u(p)) d p\right]\right\} d s-\frac{1}{\kappa} \int_{a}^{T}\left\{\left(B \alpha_{2}(T-s)+\beta_{2} \eta(t)\right)\right. \\
& \left.\times\left(\lambda_{1} f(s, u(s))+\lambda_{2} \int_{a}^{s} g(p, u(p)) d p\right)\right\} d s
\end{aligned}
$$




$$
\begin{aligned}
& +\frac{1}{\kappa} \sum_{j=1}^{m} \int_{a}^{v_{j}}\left\{( B ( v _ { j } - s ) \gamma _ { j } + \rho _ { j } \eta ( t ) ) \left(\lambda_{1} f(s, u(s))\right.\right. \\
& \left.\left.\left.+\lambda_{2} \int_{a}^{s} g(p, u(p)) d p\right)\right\} d s \mid\right\} \\
\leq & (\ell \bar{w}+N) \lambda_{1} \sup _{t \in[a, T]}\left\{\frac{(t-a)^{2}}{2}+\frac{\left|A_{1}(t-a)-A_{2}\right|}{|\kappa|}\left(\left|\beta_{3}\right| \frac{(\xi-a)^{2}}{2}\right.\right. \\
& \left.+\left|\beta_{2}\right|(T-a)+\sum_{j=1}^{m} \rho_{j}\left(v_{j}-a\right)\right)+\frac{1}{\left|A_{1}\right|}\left(\left|\alpha_{3}\right| \frac{(\xi-a)^{3}}{3 !}+\left|\alpha_{2}\right| \frac{(T-a)^{2}}{2}\right. \\
& \left.\left.+\sum_{j=1}^{m} \gamma_{j} \frac{\left(v_{j}-a\right)^{2}}{2}\right)\right\}+\left(\ell_{1} \bar{w}+N_{1}\right) \lambda_{2} \sup _{t \in[a, T]}\left\{\frac{(t-a)^{3}}{3 !}\right. \\
& +\frac{\left|A_{1}(t-a)-A_{2}\right|}{|\kappa|}\left(\left|\beta_{3}\right| \frac{(\xi-a)^{3}}{3 !}+\left|\beta_{2}\right| \frac{(T-a)^{2}}{2}+\sum_{j=1}^{m} \rho_{j} \frac{\left(v_{j}-a\right)^{2}}{2}\right) \\
& \left.+\frac{1}{\left|A_{1}\right|}\left(\left|\alpha_{3}\right| \frac{(\xi-a)^{4}}{4 !}+\left|\alpha_{2}\right| \frac{(T-a)^{3}}{3 !}+\sum_{j=1}^{m} \gamma_{j} \frac{\left(v_{j}-a\right)^{3}}{3 !}\right)\right\} \\
\leq & (\ell \bar{w}+N) \lambda_{1} Q+\left(\ell_{1} \bar{w}+N_{1}\right) \lambda_{2} \bar{Q}_{1} \leq \bar{w},
\end{aligned}
$$

where $Q$ and $\bar{Q}_{1}$ are given by (2.11) and (5.4), respectively. This shows that $\overline{\mathcal{G}} B_{\bar{w}} \subset B_{\bar{w}}$.

Next we show that the operator $\overline{\mathcal{G}}$ is a contraction. Let $u, v \in \mathcal{P}$. Then, using (2.11) and (5.4), we obtain

$$
\begin{aligned}
\|\overline{\mathcal{G}} u-\overline{\mathcal{G}} v\|= & \sup _{t \in[0, T]}|\overline{\mathcal{G}} u(t)-\overline{\mathcal{G}} v(t)| \\
\leq & \sup _{t \in[a, T]} \mid \int_{a}^{t}(t-s)\left(\lambda_{1}(f(s, u(s))-f(s, v(s)))\right. \\
& \left.+\lambda_{2} \int_{a}^{s}(g(p, u(p))-g(p, v(p))) d p\right) d s \\
& +\frac{1}{2 \kappa} \int_{a}^{\xi}\left\{\left(\left|B \alpha_{3}\right|(\xi-s)+2\left|\beta_{3} \eta(t)\right|\right)(\xi-s)\right. \\
& \left.\times\left(\lambda_{1}(f(s, u(s))-f(s, v(s)))+\lambda_{2} \int_{a}^{s}(g(p, u(p))-g(p, v(p))) d p\right)\right\} d s \\
& -\frac{1}{\kappa} \int_{a}^{T}\left\{\left(B \alpha_{2}(T-s)+\beta_{2} \eta(t)\right)\right. \\
& \left.\times\left(\lambda_{1}(f(s, u(s))-f(s, v(s)))+\lambda_{2} \int_{a}^{s}(g(p, u(p))-g(p, v(p))) d p\right)\right\} d s \\
& +\frac{1}{\kappa} \sum_{j=1}^{m} \int_{a}^{v_{j}}\left\{( B ( v _ { j } - s ) \gamma _ { j } + \rho _ { j } \eta ( t ) ) \left(\lambda_{1}(f(s, u(s))-f(s, v(s)))\right.\right. \\
& \left.\left.+\lambda_{2} \int_{a}^{s}(g(p, u(p))-g(p, v(p))) d p\right)\right\} d s \mid
\end{aligned}
$$




$$
\begin{aligned}
\leq & \|u-v\|\left\{\ell \lambda _ { 1 } \operatorname { s u p } _ { t \in [ a , T ] } \left\{\frac{(t-a)^{2}}{2}+\frac{\left|A_{1}(t-a)-A_{2}\right|}{|\kappa|}\left[\left|\beta_{3}\right| \frac{(\xi-a)^{2}}{2}\right.\right.\right. \\
& \left.+\left|\beta_{2}\right|(T-a)+\sum_{j=1}^{m} \rho_{j}\left(v_{j}-a\right)\right]+\frac{1}{\left|A_{1}\right|}\left[\left|\alpha_{3}\right| \frac{(\xi-a)^{3}}{3 !}+\left|\alpha_{2}\right| \frac{(T-a)^{2}}{2}\right. \\
& \left.\left.+\sum_{j=1}^{m} \gamma_{j} \frac{\left(v_{j}-a\right)^{2}}{2}\right]\right\}+\ell_{1} \lambda_{2} \sup _{t \in[a, T]}\left\{\frac{(t-a)^{3}}{3 !}\right. \\
& +\frac{\left|A_{1}(t-a)-A_{2}\right|}{|\kappa|}\left[\left|\beta_{3}\right| \frac{(\xi-a)^{3}}{3 !}+\left|\beta_{2}\right| \frac{(T-a)^{2}}{2}+\sum_{j=1}^{m} \rho_{j} \frac{\left(v_{j}-a\right)^{2}}{2}\right] \\
& \left.\left.+\frac{1}{\left|A_{1}\right|}\left[\left|\alpha_{3}\right| \frac{(\xi-a)^{4}}{4 !}+\left|\alpha_{2}\right| \frac{(T-a)^{3}}{3 !}+\sum_{j=1}^{m} \gamma_{j} \frac{\left(v_{j}-a\right)^{3}}{3 !}\right]\right\}\right\} \\
\leq & \left\{\ell \lambda_{1} Q+\ell_{1} \lambda_{2} \bar{Q}_{1}\right\}\|u-v\|,
\end{aligned}
$$

which, in view of condition (5.5), implies that the operator $\overline{\mathcal{G}}$ is a contraction. Thus, by Banach's contraction mapping principle, we deduce that the operator $\overline{\mathcal{G}}$ has a unique fixed point, which corresponds to a unique solution of problem (5.1) on $[a, T]$.

Example 5.2 Consider the following second-order integro-differential boundary value problem:

$$
\left\{\begin{array}{l}
u^{\prime \prime}(t)=\lambda_{1} f(t, u(t))+\lambda_{2} \int_{a}^{t} g(s, u(s)) d s, \quad t \in[1,2], \\
\alpha_{1} u(a)+\alpha_{2} u(T)=\alpha_{3} \int_{a}^{\xi} u(s) d s+\sum_{j=1}^{4} \gamma_{j} u\left(v_{j}\right), \\
\beta_{1} u^{\prime}(a)+\beta_{2} u^{\prime}(T)=\beta_{3} \int_{a}^{\xi} u^{\prime}(s) d s+\sum_{j=1}^{4} \rho_{j} u^{\prime}\left(v_{j}\right),
\end{array}\right.
$$

where $f(t, u(t))$ and all the constants $\xi, \alpha_{i}, \beta_{i}(i=1,2,3), \gamma_{j}, v_{j}, \rho_{j}(j=1, \ldots, 4)$ are the same as in Example 2.10. Further, we take $\lambda_{1}=1, \lambda_{2}=1$, and

$$
g(t, u(t))=\frac{4}{7 \sqrt{t^{2}+3}} \tan ^{-1} u(t)+\frac{1}{5} e^{-t}
$$

which satisfies $\left(H_{5}\right)$ with $\ell_{1}=\frac{2}{7}$. Further, we obtain $\bar{Q}_{1}=0.479676\left(\bar{Q}_{1}\right.$ is defined by (5.4)) and $\ell \lambda_{1} Q+\ell_{1} \lambda_{2} \bar{Q}_{1} \approx 0.664995<1$. Clearly all the conditions of Theorem 5.1 are satisfied. Hence it follows by Theorem 5.1 that there exists a unique solution for problem (5.6) on $[1,2]$.

Remark 5.3 Existence results analogous to the ones for problem (1.1) can be obtained for problem (5.1) in a similar manner.

\section{Conclusions}

We have discussed the existence and uniqueness of solutions and Ulam stability for second-order boundary value problems involving nonlocal non-separated type integral multi-point boundary conditions on an arbitrary domain. An associated boundary value problem involving integro-differential equations is also investigated. Our results are not only new in the given configuration, but also specialize to several results by fixing the values of parameters and constants involved in the problems. We believe that the present 
work is a useful contribution to the existing literature on the second-order boundary value problems.

\section{Funding}

Not applicable.

Abbreviations

Not applicable.

Availability of data and materials

Not applicable.

\section{Competing interests}

The authors declare that they have no competing interests.

\section{Authors' contributions}

Each of the authors, AA, MA, RPA, and BA contributed equally to each part of this work. All authors read and approved the final manuscript.

\section{Author details}

${ }^{1}$ Nonlinear Analysis and Applied Mathematics (NAAM) — Research Group, Department of Mathematics, Faculty of Science, King Abdulaziz University, Jeddah, Saudi Arabia. ${ }^{2}$ Department of Mathematics, Texas A\&M University, Kingsville, USA.

${ }^{3}$ Florida Institute of Technology, Melbourne, USA.

\section{Publisher's Note}

Springer Nature remains neutral with regard to jurisdictional claims in published maps and institutional affiliations.

Received: 15 May 2018 Accepted: 18 June 2018 Published online: 28 June 2018

\section{References}

1. Bressan, A.: Hyperbolic Systems of Conservation Laws. The One-dimensional Cauchy Problem. Oxford University Press, Oxford (2000)

2. Polyanin, A.D., Zaitsev, V.F.: Handbook of Nonlinear Partial Differential Equations. Chapman \& Hall/CRC, Boca Raton (2004)

3. Akyildiz, F.T., Bellout, H., Vajravelu, K., Van Gorder, R.A.: Existence results for third order nonlinear boundary value problems arising in nano boundary layer fluid flows over stretching surfaces. Nonlinear Anal., Real World Appl. 12 2919-2930 (2011)

4. Zheng, L., Zhang, X:: Modeling and Analysis of Modern Fluid Problems. Mathematics in Science and Engineering. Academic Press, London (2017)

5. Greguš, M., Neumann, F., Arscott, F.M.: Three-point boundary value problems in differential equations. Proc. Lond. Math. Soc. 3, 459-470 (1964)

6. Bicadze, A.V., Samarskii, A.A.: Some elementary generalizations of linear elliptic boundary value problems. Dokl. Akad. Nauk SSSR 185, 739-740 (1969) (Russian)

7. Il'in, V.A., Moiseev, E.I.: Nonlocal boundary-value problem of the first kind for a Sturm-Liouville operator in its differential and finite difference aspects. Differ. Equ. 23, 803-810 (1987)

8. Andres, J.: A four-point boundary value problem for the second-order ordinary differential equations. Arch. Math. (Basel) 53, 384-389 (1989)

9. Gupta, C.P.: Solvability of a three-point nonlinear boundary value problem for a second order ordinary differential equations. J. Math. Anal. Appl. 168, 540-551 (1998)

10. Eloe, P.W., Ahmad, B.: Positive solutions of a nonlinear $n$th order boundary value problem with nonlocal conditions. Appl. Math. Lett. 18, 521-527 (2005)

11. Clark, S., Henderson, J.: Uniqueness implies existence and uniqueness criterion for non local boundary value problems for third-order differential equations. Proc. Am. Math. Soc. 134, 3363-3372 (2006)

12. Webb, J.R.L., Infante, G.: Positive solutions of nonlocal boundary value problems: a unified approach. J. Lond. Math. Soc. 74, 673-693 (2006)

13. Ma, R.: A survey on nonlocal boundary value problems. Appl. Math. E-Notes 7, 257-279 (2007)

14. Graef, J.R., Webb, J.R.L.: Third order boundary value problems with nonlocal boundary conditions. Nonlinear Anal. 71, 1542-1551 (2009)

15. Wang, L., Pei, M., Ge, W.: Existence and approximation of solutions for nonlinear second-order four-point boundary value problems. Math. Comput. Model. 50, 1348-1359 (2009)

16. Sun, Y., Liu, L., Zhang, J., Agarwal, R.P.: Positive solutions of singular three-point boundary value problems for second-order differential equations. J. Comput. Appl. Math. 230, 738-750 (2009)

17. Feng, M., Zhang, X., Ge, W.: Existence theorems for a second order nonlinear differential equation with nonlocal boundary conditions and their applications. J. Appl. Math. Comput. 33, 137-153 (2010)

18. Ashordia, M.: On boundary value problems for systems of nonlinear generalized ordinary differential equations. Czechoslov. Math. J. 67, 579-608 (2017)

19. Liu, R., Wu, Z:: Well-posedness of a class of two-point boundary value problems associated with ordinary differential equations. Adv. Differ. Equ. 2018, Article ID 54 (2018) 
20. Taylor, C., Hughes, T., Zarins, C.: Finite element modeling of blood flow in arteries. Comput. Methods Appl. Mech. Eng. 158, 155-196 (1998)

21. Womersley, J.R.: Method for the calculation of velocity, rate of flow and viscous drag in arteries when the pressure gradient is known. J. Physiol. 127, 553-563 (1955)

22. Nicoud, F., Schfonfeld, T.: Integral boundary conditions for unsteady biomedical CFD applications. Int. J. Numer. Methods Fluids 40, 457-465 (2002)

23. Cannon, J.R.: The solution of the heat equation subject to the specification of energy. Q. Appl. Math. 21, 155-160 (1963)

24. Ionkin, N.I.: The solution of a certain boundary value problem of the theory of heat conduction with a nonclassical boundary condition. Differ. Uravn. 13, 294-304 (1977) (Russian)

25. Chegis, R.Yu: Numerical solution of a heat conduction problem with an integral condition. Liet. Mat. Rink. 24, 209-215 (1984)

26. Webb, J.R.L., Infante, G.: Positive solutions of nonlocal boundary value problems involving integral conditions. NoDEA Nonlinear Differ. Equ. Appl. 15, 45-67 (2008)

27. Ahmad, B., Alsaedi, A.: Existence of approximate solutions of the forced Duffing equation with discontinuous type integral boundary conditions. Nonlinear Anal., Real World Appl. 10, 358-367 (2009)

28. Boucherif, A.: Second-order boundary value problems with integral boundary conditions. Nonlinear Anal., Theory Methods Appl. 70, 364-371 (2009)

29. Ahmad, B., Ntouyas, S.K., Alsulami, H.H.: Existence results for $n$-th order multipoint integral boundary-value problems of differential inclusions. Electron. J. Differ. Equ. 2013, Article ID 203 (2013)

30. Henderson, J.: Smoothness of solutions with respect to multi-strip integral boundary conditions for $n$th order ordinary differential equations. Nonlinear Anal., Model. Control 19, 396-412 (2014)

31. Karaca, I.Y., Fen, F.T.: Positive solutions of $n$ th-order boundary value problems with integral boundary conditions. Math. Model. Anal. 20, 188-204 (2015)

32. Ahmad, B., Alsaedi, A., Al-Malki, N.: On higher-order nonlinear boundary value problems with nonlocal multipoint integral boundary conditions. Lith. Math. J. 56, 143-163 (2016)

33. Boukrouche, M., Tarzia, D.A.: A family of singular ordinary differential equations of the third order with an integral boundary condition. Bound. Value Probl. 2018, Article ID 32 (2018)

34. Smart, D.R.: Fixed Point Theorems. Cambridge Tracts in Mathematics. Cambridge University Press, Cambridge (2005)

35. Granas, A., Dugundji, J.: Fixed Point Theory. Springer, New York (2005)

\section{Submit your manuscript to a SpringerOpen ${ }^{\circ}$ journal and benefit from:}

- Convenient online submission

- Rigorous peer review

- Open access: articles freely available online

- High visibility within the field

- Retaining the copyright to your article

Submit your next manuscript at $\boldsymbol{~ s p r i n g e r o p e n . c o m ~}$ 